\title{
Vielfalt, die verbindet: Der Übergang Schule-Hochschule im Rahmen des Hanse-Kolloquiums zur Hochschuldidaktik der Mathematik 2018 in Essen
}

\author{
Klinger, Marcel $^{1}$; Schüler-Meyer, Alexander ${ }^{2}$; Wessel, Lena ${ }^{3}$
}

${ }^{1}$ Universität Duisburg-Essen, Essen; ${ }^{2}$ Technische Universität Eindhoven; ${ }^{3}$ Pädagogische Hochschule Freiburg

\section{Ausgangslage}

Die universitäre Lehre in der Mathematik steht derzeit gerade im Bereich des Übergangs zwischen Schule und Hochschule im Fokus vieler Innovationen. Dies spiegelt sich auch in der Vielfalt der Beiträge und Akteure im Rahmen des Hanse-Kolloquium zur Hochschuldidaktik der Mathematik wider, welches vom 9. bis 10. November 2018 als gemeinsame Herbsttagung mit dem Arbeitskreis HochschulMathematikDidaktik der GDM an der Universität Duisburg-Essen stattfand. Die Gründe für diese Innovationen liegen in vielfältigen Bereichen, die erst seit wenigen Jahren wahrgenommen werden:

Heterogenität der fachlichen Voraussetzungen der Studierenden: Abhängig davon, wo Jugendliche ihre Hochschulzugangsberechtigung erworben haben, sind sie in den letzten zwei Schuljahren vor der Abiturprüfung in sehr unterschiedlichen Mathematikkursvarianten auf das Mathematikstudium bzw. mathematikhaltige Veranstaltungen anderer Studiengänge vorbereitet worden. So ist beispielsweise in fünf Bundesländern Mathematik als Prüfungsfach für das Abitur verpflichtend, in allen anderen Bundesländern muss Mathematik zwar in zwei- bis sechsstündigen Kursen belegt werden, aber nicht als Prüfungsfach.

Hohe Abbruchquoten: Im Wintersemester 2017/2018 waren mindestens $55 \%$ aller Studierenden in der Bundesrepublik in Fächern eingeschrieben, in denen mindestens ein mathematisches Modul im Laufe des Studiums belegt werden muss. Gleichzeitig liegen die Abbruchquoten in MINT-Studienfächern im Absolventenjahrgang 2018 mit $35 \%$ (Ingenieurwesen) bis $54 \%$ (Mathematik) deutlich über dem bundesweiten Durchschnitt von $28 \%$. (Heublein et al., 2012)

Die sich ändernde Natur des mathematischen Denkens und Arbeitens: Die Art des mathematischen Denkens ändert sich von der Schule zur Hochschule. So wird beispielsweise mehr Wert auf deduktives Schlussfolgern gelegt, und generell spielen das Beweisen und Definieren eine zentralere Rolle in der Erkenntnisgewinnung (Schüler-Meyer \& Rach, 2019).

Trotz der Vielfältigkeit der genannten Phänomene zeigt sich aber doch, dass Lehrende an Hochschulen passende Erwartungen haben: Die MaLeMINT-Studie des IPN in Kiel 
konnte zeigen, dass es einen ,sehr breiten Konsens unter Hochschullehrenden der Mathematik hinsichtlich der Lernvoraussetzungen“ der Studierenden in MINT-Fächern gibt. Die Erwartungen der Hochschullehrenden sind dabei ,in weiten Teilen anschlussfähig an das, was in der Regel in schulischen Mathematikcurricula abgedeckt wird“" (Pigge, Neumann \& Heinze, 2019, S. 36).

In Reaktion auf diese und weitere Herausforderungen wurden und werden vielfältige Änderungen umgesetzt und erprobt. So sind Übergangskurse nahezu fester Bestandteil universitärer Lehrangebote. Diese Übergangskurse zielen oft auf eine Wiederholdung des Schulstoffs in der Hoffnung, alle Studierenden auf den gleichen Vorwissensstand zu bringen. Andere Brückenkurse zielen eher auf die Vorbereitung auf das für Lernende neuartige mathematische Denken, entweder an der Universität (Grieser, 2017) oder bereits in der schulischen Oberstufe (Heitzer, 2019; Schüler-Meyer, 2019). In diesem Zuge sind viele Dozierende an Hochschulen bereit, neuartige Wege bei der Verbesserung der mathematischen Lehre zu gehen:

\section{Reichhaltigkeit der Zugänge zur Lösung der Übergangsproblematik}

Der Übergang von der Schule zur Hochschule wird aus verschiedenen Perspektiven und durch verschiedene Zugänge andressiert und bearbeitet. Die Beiträge zum Hanse-Kolloquium in diesem Band bilden diese Perspektiven in ihrer Vielfältigkeit ab.

- Hochschuldidaktische Ansätze nehmen die Übergangsproblematik aus der Perspektive der spezifischen universitären Lehr-Lernsituationen in den Blick, die sich von denen der Schule unterscheiden. Hochschulmathematikdidaktische Ansätze richten ihren Blick auf die Spezifität des Mathematiklernens im Übergang, wie etwa die Problematik, dass Schulwissen Studierenden im Studium zwar wiederbegegnet, aber auf nicht vertraute Weise, die zu nicht tragfähigen mathematischem Denken führen kann (Nardi, Ryve, Stadler \& Viirman, 2014).

- Es gibt eine große Heterogenität bzgl. der Zielgruppe, auf die sich hochschulmathematikdidaktische Forschung und Entwicklung bezieht: Lehramt mit jeweiligem Schulschwerpunkt (G H R Ge Gy BK SoPäd), Fachbachelor/Master Mathematik, polyvalenter Bachelor (Mathematik-Bachelor mit Lehramtsoption), Studiengänge mit hohen mathematischen Studienanteilen in den Bereichen der Ingenieurwissenschaften, Wirtschaftswissenschaften usw., oft auch verschlagwortet als Export- oder Service-Veranstaltungen).

- Es gibt viele Best-Practice-Beispiele von Innovationen in der Lehre, die zu innovativen Begleitforschungen einladen - was allerdings vom Zusammenfinden von Forschern und Praktikern lebt.

- Der Druck, die Lehre zu innovieren, führt zu verschiedensten Lehrangeboten. Es besteht jedoch großer Bedarf, ähnliche Angebote zu kombinieren, voneinander zu lernen und so gemeinsam an der Optimierung dieser Angebote zu arbeiten.

- Das Feld profitiert von der Vielfältigkeit der Perspektiven, die zusammenkommen, um die Übergangsproblematik zu bearbeiten. Mathematiker gehen auf die Mathematikdidaktik zu, Mathematikdidaktiker spezialisieren sich zunehmend auf das Mathematiklehren und -lernen an der Hochschule. Dozierende an Fachhochschulen bilden einen weiteren lebendigen Teil dieser Dynamik ab. 
- Neue Medien erlauben neuartige Lehr- und Lernformen und eröffnen zugleich neue Ressourcen für die Studierenden (wie YouTube-Videos, Lernplattformen oder mobile Apps). Digitale Ressourcen werden von Studierenden intensiv genutzt und sind beliebt (Rat für Kulturelle Bildung, 2019). Sie erzeugen aber auch das Risiko einer „Verstehensillusion“, bei der Lernende oder Lehrende glauben, durch das alleinige Rezipieren von Videos könne mathematisches Verständnis erworben werden (z. B. Kulgemeyer, 2018).

Insgesamt illustrieren diese Punkte eine Intensivierung von Lehr-Lern-Entwicklung und Forschungsaktivitäten an der Schnittstelle Schule-Hochschule. Während große Potentiale vorhanden sind, Ressourcen und Perspektiven zu bündeln, um den Übergang von der Schule zur Hochschule zu erleichtern, werden diese noch nicht immer genutzt.

\section{Das Potential des Hanse-Kolloquiums als Inkubator für Ideen und Koope- rationen über Institutionsgrenzen hinweg}

Der aufmerksame Zuhörer kann auf dem Hanse-Kolloquium noch immer Fachgrenzen wahrnehmen und unterschiedliche Überzeugungen und Perspektiven ausmachen. Dabei sind diese persönlichen Überzeugungen und Perspektiven Ausdruck der unterschiedlichen Bedarfe und Besonderheiten, die an den jeweiligen Institutionen wirken, wie etwa die besonders problematische Situation an Fachhochschulen, mit wenigen verfügbaren Ressourcen einer extrem heterogenen Studierendenschaft gerecht zu werden. Oder die Unterschiedlichkeiten, die im Hinblick auf das mathematische Denken und Arbeiten vorliegen, an welchem Studierende teilhaben sollen.

Dem Hanse-Kolloquium gelingt es seit seinem Bestehen immer wieder, Akteure und Interessierte im Bereich der Hochschulmathematik zusammen zu bringen, d. h. Lehrende und Dozierende an Universitäten und Fachhochschulen, Forschende im Bereich des tertiären Mathematiklernens, sowie wissenschaftlichen Nachwuchs als auch Professorinnen und Professoren. Auch wenn sich das Hanse-Kolloquium im Jahre 2018 das Thema „Übergang Schule-Hochschule“ gesetzt hat, zeigt diese Vielfältigkeit doch auf, dass es den Übergang nicht gibt und geben kann. Der Übergang von der Schule zur Hochschule ist aus Sicht der verschiedenen Institutionen unterschiedlich zu konzipieren. Auch aus Sicht der Fächer gibt es nicht den Übergang: die Studierenden in den Lehramtsfächern unterscheiden sich von jenen der Fachmathematik, die sich wiederum von Studierenden der Ingenieurswissenschaften, etc. unterscheiden. Weitere Unterschiede finden sich in den verschiedenen Lernvoraussetzungen, Lernbiografien, Erwartungen, Interessen usw. der Studierenden.

Die komplexe Topografie des Mathematiklernens im Übergang führt zu unterschiedlichen Herausforderungen. Aus den Beiträgen des Hanse-Kolloquiums können u. a. die folgenden Herausforderungen identifiziert werden:

- Eine extrem heterogene Studierendenschaft hinsichtlich der Bildungsbiografien (z. B. langjährig im Beruf tätige Personen, die ein Studium ergreifen), die verschiedenes, teilweise, verschüttetes ' mathematisches Vorwissen mitbringt. Diese Studierenden sollen mit wenigen Ressourcen auf den gleichen mathematischen Wissensstand gebracht werden. Dies führt zu Überlegungen hinsichtlich des Einsatzes digitaler (Selbst-)Lerntools und Lernvideos sowie zur Konzeption von 
Flipped-Classroom-Formaten (vgl. die Beiträge zu den Hauptvorträgen von Frode Rønning bzw. Bärbel Barzel).

- Studierende sind mit hochschulmathematischen Denk- und Arbeitsweisen nur wenig vertraut, nutzen ineffiziente Lernstrategien und müssen diese für die Hochschule und insbesondere für das arbeitsintensive mathematikhaltige Studium verändern. Unterstützung können Studierende dafür in hochschuleigenen Förderangeboten wahrnehmen. Dies führt zu individuellen Förderangeboten, aber auch zu Angeboten, bei denen ein direkterer Dozenten-Studierendenkontakt möglich ist. Exemplarisch werden in diesem Band entsprechende Konzepte der Technischen Universität sowie Fachhochschule Dortmunds vorgestellt (vgl. Beitrag zum Hauptvortrag von Nimet Sarikaya und Peter Furlan).

Die Vielfältigkeit der Herausforderungen des Übergangs zu einem mathematikhaltigen Studium führen zu vielfältigen Versuchen, diesen Herausforderungen zu begegnen. Beim Hanse-Kolloquium kommen Teilnehmerinnen und Teilnehmer aus allen Disziplinen zusammen, um sich über innovative Ansätze und Praxiserfahrungen auszutauschen. Dabei ordnen sich die Beiträge auf einem Spektrum von Grundlagenforschung (Was ist die genaue Natur des Übergangs Schule-Hochschule?) über forschungsbasierte Praxisversuche (Kann eine Vorlesung die eigenaktive Begriffsbildung bei Studierenden unterstuitzen?) hin zu breiter implementierten und evaluierten Maßnahmen (Wie werden implementierte Unterstützungmaßnahmen genutzt?) ein. Allen diesen Beiträgen ist die Suche nach Evidenz, wie den Herausforderungen in der mathematischen Lehre in Hochschulen wirkungsvoll begegnet werden kann, gemein. Insgesamt zeigt sich, dass sich Lehrinnovationen und Forschungsaktivitäten in den Hochschulen in den letzten Jahren intensiviert haben, nicht nur an der Schnittstelle Schule-Hochschule, sondern auch grundsätzlicher bezogen auf hochschulmathematikdidaktische Forschung und Entwicklung.

Wir wünschen vielfältige Einblicke bei der Lektüre des vorliegenden Bands.

Essen, Juli 2019

Marcel Klinger

Alexander Schüler-Meyer

Lena Wessel 


\section{Literatur}

Grieser, D. (2017). Mathematisches Problemlösen und Beweisen: Eine Entdeckungsreise in die Mathematik (2. Aufl.). Wiesbaden: Springer Spektrum.

Heitzer, J. (2019). Erfolgreiche Vorbereitung auf mathematische Studienanforderungen in der Oberstufe: Das Aachener Schul-Hochschul-Projekt iMPACt. Der Mathematikunterricht, 65(2), 20-28.

Heublein, U., Richter, J., Schmelzer, R., \& Sommer, D. (2012). Die Entwicklung der Schwundund Studienabbruchquoten an den deutschen Hochschulen. Statistische Berechnungen auf der Basis des Absolventenjahrgangs 2010. Hannover: HIS.

Kulgemeyer, C. (2018). Wie gut erklären Erklärvideos? Ein Bewertungs-Leitfaden. Computer + Unterricht, Heft 109, 8-11.

Nardi, E., Ryve, A., Stadler, E., \& Viirman, O. (2014). Commognitive analyses of the learning and teaching of mathematics at university level: the case of discursive shifts in the study of calculus. Research in Mathematics Education, 16(2), 182-198.

Pigge, C., Neuman, I., \& Heinze, A. (2019). Notwendige mathematische Lernvoraussetzungen für MINT-Studiengänge - die Sicht der Hochschullehrenden. Der Mathematikunterricht, 65(2), 29-38.

Rat für Kulturelle Bildung (2019). Jugend / YouTube / Kulturelle Bildung: Horizont 2019. Essen: Rat für Kulturelle Bildung.

Schüler-Meyer, A. \& Rach, S. (Hrsg.) (2019). Der Übergang vom Mathematikunterricht in ein MINT-Studium: Herausforderungen und Unterstützungsansätze [Themenheft]. Der Mathematikunterricht, 65(2).

Schüler-Meyer, A. (2019). Hochschulmathematische Praktiken an schulnahen Inhalten - Ein tragfähiges Gestaltungsprinzip für Brückenkurse in der schulischen Oberstufe. Der Mathematikunterricht, 65(2), 47-55. 


\section{DuEPublico}

UNIVERSITÄT
DEUSSSEN R G

offen im Denken

\section{Duisburg-Essen Publications online}

Dieser Text wird über DuEPublico, dem Dokumenten- und Publikationsserver der Universität Duisburg-Essen, zur Verfügung gestellt. Die hier veröffentlichte Version der EPublikation kann von einer eventuell ebenfalls veröffentlichten Verlagsversion abweichen.

DOI: $\quad 10.37626 / \mathrm{GA} 9783959870986.0 .01$

URN: urn:nbn:de:hbz:464-20210202-174959-9

Klinger, M., Schüler-Meyer, A., Wessel, L. (2019). Vielfalt, die verbindet: Der Übergang SchuleHochschule im Rahmen des Hanse-Kolloquiums zur Hochschuldidaktik der Mathematik 2018 in Essen. Erschienen in: M. Klinger, A. Schüler-Meyer \& L. Wessel (Hrsg.), Hanse-Kolloquium zur Hochschuldidaktik der Mathematik 2018: Beiträge zum gleichnamigen Symposium am 9. \& 10. November 2018 an der Universität Duisburg-Essen. Münster: WTM-Verlag, 2019. S. 3-8. DOI: https://doi.org/10.37626/GA9783959870986.0.01

Alle Rechte vorbehalten. 